

\section{REVISTA \\ TEORÍA Y PRÁCTICA \\ DE LA \\ ARQUEOLOGÍA HISTÓRICA LATINOAMERICANA}

ISSN: 2250-866X (impreso) | ISSN: 2591-2801 (en línea)

AÑO VIII, VOLUMEN 9, PRIMAVERA DE 2019

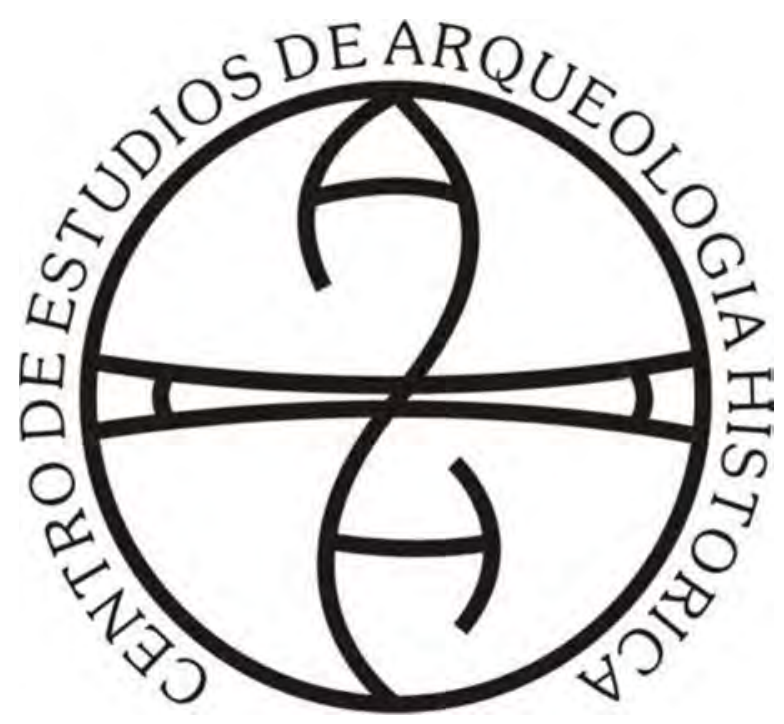

CENTRO de Estudios de ARqueOlOGía HistóricA

FACULTAD DE HUMANIDADES Y ARTES | UNIVERSIDAD NACIONAL DE ROSARIO 
(Universidad Nacional de Rosario, Universidad Nacional de Río Cuarto,

Universidad Nacional de San Juan, Universidad de la República, Universidad Nacional de Trujillo)

\author{
AUTORIDADES DE LA UNIVERSIDAD NACIONAL DE ROSARIO \\ RECTOR: Lic. Franco Bartolacci \\ VICE-RECTOR: Od. Darío Macía \\ SECRETARIO GENERAL: Prof. José Goity \\ SECRETARIA ACADÉMICO Y DE APRENDIZAJE: Dr. Marcelo Vedrovnik \\ SECRETARÍA DE CIENCIA TECNOLOGÍA E INNOVACIÓN \\ PARA EL DESARROLLO: Ing. Guillermo Montero.
}

\author{
AUTORIDADES DE LA FACULTAD DE HUMANIDADES Y ARTES \\ DECANO: Prof. Alejandro Vila \\ VICEDECANA: Prof. Marta Varela \\ SECRETARIA ACADÉMICA: Dra. Marcela Coria
}

\author{
AUTORIDADES DEL CENTRO DE ARQUEOLOGÍA HISTÓRICA \\ DIRECTORA: Dra. Ana Rocchietti \\ SECRETARIA: Prof. Nélida de Grandis \\ PROSECRETARIA: Lic. Marianela Bizcaldi
}

DIRECTORAS - EDITORAS:

Dra. Ana Rocchietti y Prof. Nélida De Grandis

SECRETARIA DE EDICIÓN: Dra. Irene Dosztal

Este número es co-edición de las ponencias

del VIII Congreso Nacional de Arqueología Histórica (2018) entre:

Centro de Estudios en Arqueología Histórica: Directora Ana Rocchietti

Centro de Estudios en Arqueología Regional: Director Fernando Oliva

Centro de Estudios en Arqueología Subacuática: Directora Mónica Valentini

Departamento de Arqueología, Escuela de Antropología: Director Fernando Oliva
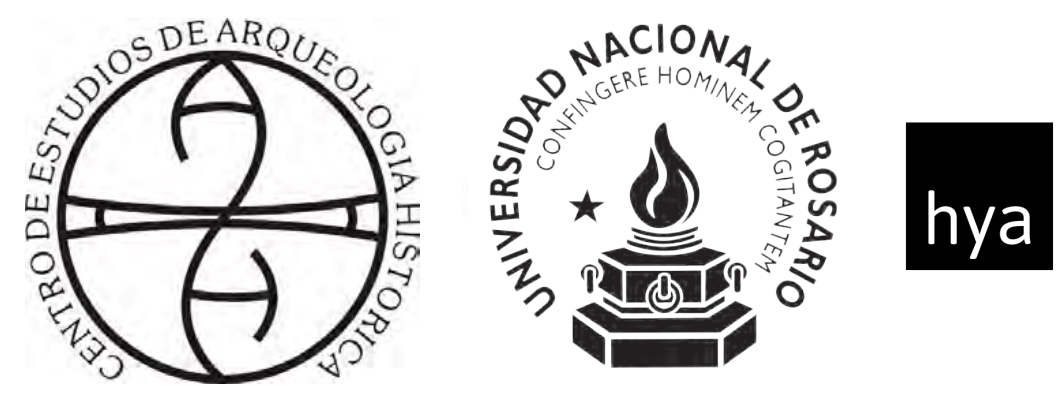

Facultad de Humanidades

y Artes_UNR
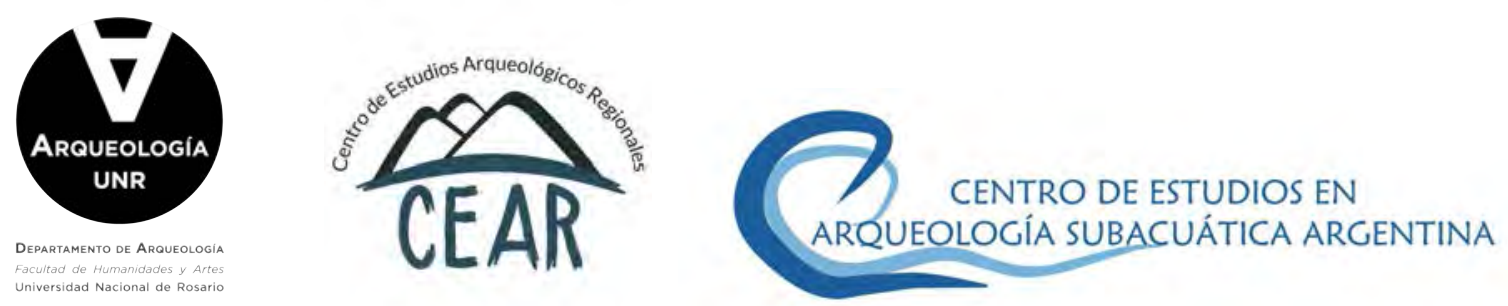
Comité Permanente de los Congresos Nacionales de Arqueología Histórica

Dr. Daniel Schávelzon (Universidad Nacional de

Buenos Aires)

Prof. María Teresa Carrara (Universidad Nacional de Rosario)

Prof. Carlos Baldassarre (Museo Municipal de Río

Grande, Tierra del Fuego) in memoriam

Dr. Mariano Ramos (Universidad Nacional de Luján, CONICET)

Dr. Horacio Chiavazza (Universidad Nacional de Cuyo)

Dra. Ana María Rocchietti (Universidad Nacional de

Rosario, Universidad Nacional de Río Cuarto)

Lic. Facundo Gómez Romero (Universidad Autónoma

de Barcelona)

\section{Comité Científico}

Dra. Tânia Andrade Lima (Universidade Federal do Rio de Janeiro)

Prof. Réginald Auger (CELAT/Département des

Sciences Historiques, Université Laval, Canadá)

Dr. Roberto Bárcena (Universidad Nacional de Cuyo, CONICET)

Dra. Marta Bonaudo (Universidad Nacional de Rosario, CONICET)

Dr. Leonel Cabrera (Universidad de la República, Uruguay)

Dr. Luis María Calvo (Universidad Católica de Santa

$\mathrm{Fe})$

Prof. Juan Castañeda Murga (Universidad Nacional de

Trujillo, Perú)

Dr. Carlos Ceruti (Museo de Ciencias Naturales y

Antropología "Prof. Antonio Serrano”. Paraná)

Dr. Horacio Chiavazza (Universidad Nacional de Cuyo)

Dra. Silvia Cornero (Universidad Nacional de Rosario)

Prof. Pedro Paulo Funari (Universidade Estadual de

Campinas, Brasil)

Lic. Jorge A. Gamboa Velásquez (Universidad Nacional

Santiago Antuñez de Mayolo, Perú)

Dr. Eduardo Alejandro García (Universidad Nacional de

San Juan, CONICET)

Prof. Nélida De Grandis (Universidad Nacional de

Rosario)

Dr. Juan Bautista Leoni (Universidad Nacional de

Rosario, CONICET)

Dra. Amancay Martínez (Universidad Nacional de San

Luis)

Dra. Catalina Teresa Michieli (Universidad Nacional de

San Juan, CONICET)

Lic. Fernando Oliva (Universidad Nacional de Rosario)
Ing. Adrián Pifferetti (Universidad Tecnológica Nacional Regional Rosario)

Dr. Mariano Ramos (Universidad Nacional de Luján, CONICET)

Dra. Ana María Rocchietti (Universidad Nacional de Rosario)

Dr. Daniel Schávelzon, (Universidad Nacional de Buenos Aires, CONICET)

Dra. Carlota Sempé (Universidad Nacional de La Plata)

Dr. Mario Silveira (Universidad Nacional de Buenos

Aires)

Dra. Silvia Simonassi (Universidad Nacional de Rosario)

Dra. Alicia Tapia (Universidad Nacional de Buenos Aires, Universidad Nacional de Luján)

Lic. Mónica P. Valentini (Universidad Nacional de Rosario)

Agrim. Benito Vicioso (Universidad Nacional de Rosario)

\section{Evaluaron este volumen}

Roberto Bárcena, María Teresa Boschin, Leonel Cabrera, Ulises Camino, María Rosa Carbonari, Carlos Ceruti, Horacio Chiavazza, Nicolás Ciarlo, Silvia Cornero, Eduardo Crivelli, Javier García Cano, Martín Gentinetta, María Laura Gili, Carlos Landa, Matilde Lanza, Melina Malandrino, Sebastián Pastor, Victoria Pedrotta, Josefina Piana, Mercedes Podestá, Mariano Ramos, Daniel Schavelzon, Diana Tamburini, Mónica Therrien, Mónica Valentini y María Teresa Boschin

\section{Diseño y diagramación}

Eugenia Reboiro

(eugenia.reboiro@gmail.com)

\section{Curadoría}

Ana Rocchietti e Irene Dosztal

Foto de tapa: Material arqueológico, del texto de Tapia et al.

\section{Propietario responsable:}

Facultad de Humanidades y Artes, Universidad Nacional de Rosario Centro de Estudios de Arqueología Histórica

Entre Ríos 758. Rosario, provincia de Santa

Fe (2000). Argentina.

Telf.: +54 (0341) 4802670

E-mail: ceahunr@gmail.com

Decreto Ley 6422/57 de Publicaciones

Periódicas 


\section{Índice}

Editorial

El origen del patrimonio histórico y su conservación integral en la contemporaneidad.

Yanina Aguilar

Prospección de basurales históricos de la ciudad de Rosario.

Soccorso Volpe y Gustavo Fernetti

Desconstrucción de un paisaje urbano. El Montevideo del extramuros colonial, aportes de la arqueología a su identidad actual. Ana Gamas

La Calota de Ameghino: reconsiderando un viejo tema desde la arqueología histórica.

Daniel Schávelzon

Análisis zooarqueológico de los restos hallados en una estructura sanitaria vinculada a momentos tempranos del actual barrio de Belgrano .71 Mario Silveira, Horacio Padula, Ricardo Orsini y Eva Bernat

La medida del tiempo: el reloj solar jesuita de La Cruz, provincia de Corrientes.

Fernando Oliva y María Cecilia Panizza

Sitio La Quinta: arqueología rural de campos serranos.

Flavio Ribero

Si no hay tabaco que no se note. Prácticas fumatorias en el fortin La Perra (1883-1885), La Pampa.

Alicia Tapia, Virginia Pineau y Melisa Ayelén Auge

El uso del tabaco y las pipas de caolin (clay pipes) en la frontera sur y oeste de la provincia de Buenos Aires en el siglo XIX.. .127

María del Carmen Langiano y Julio Fabián Merlo 
Patrimonio natural, urbano y arquitectónico de la costa en Mar del Plata. Federico Negroni

Huellas urbanas.

Ezequiel Serrot

Paisaje y patrimonio. La industria taninera en el siglo XX.

Provincia de Santa Fe.

Cristina Pasquali, Paola Milicic y Lara Ferré

Los desafíos de las arqueologías históricas latinoamericanas.

Andrés Zarankin

Sellos entre escombrales. Las lozas en el registro urbano

del Área Fundacional de Mendoza (siglo XIX y principios del siglo XX)

Lorena Puebla y Horacio Chiavazza

Evolución edilicia de la Plaza de Mayo de la Ciudad

de Buenos Aires (1580-1853).

Nicolás Ferrino

Gestión patrimonial en el rescate arqueológico y futuro Museo de Sitio

en Moreno 550, Ciudad Autónoma de Buenos Aires, Argentina.

María Eva Bernat, Ricardo Orsini, Horacio Padula y Mario Silvera 


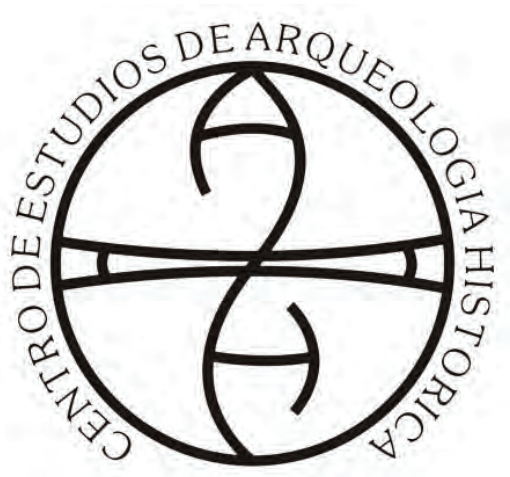

Centro de Estudios de Arqueología Histórica

Universidad Nacional de Rosario
Teoría y Práctica de la Arqueología Histórica

Latinoamericana | Año VIII. Vol. 9 | 2019

Revista del Centro de Estudios de Arqueología

Histórica, Facultad de Humanidades y Artes,

Universidad Nacional de Rosario

https://teoriaypracticaah.unr.edu.ar/index.php/index

ceahunr@gmail.com

ISSN en línea: 2591-2801

ISSN versión impresa: 2250-866X

\title{
Si no hay tabaco Que no se note. Prácticas fumatorias En el fortin La Perra (1883-1885), La Pampa
}

\author{
Alicia Tapia*, Virginia Pineau** y Melisa Ayelén Auge***
}

\section{Resumen}

En este artículo se presentan los resultados obtenidos a partir del análisis de microrrestos vegetales identificados en una pipa artesanal hallada en el Fortín La Perra, departamento Loventue, La Pampa. En dicho sitio se instaló un pequeño asentamiento militar que formó parte de una línea de fortines planteada a partir de 1882, como estrategia para ejercer la vigilancia y el control del territorio del cual fueron expulsados los ranqueles durante la Conquista del Desierto en 1879. El patrón de asentamiento de este sitio así como los materiales vítreos, faunísticos y de metal recuperados han sido objeto de diferentes estudios ya publicados. En este caso, el estudio específico del contenido de la pipa constituye un nuevo aporte a los conocimientos alcanzados sobre la falta de provisiones y el aislamiento al que fueron sometidos los integrantes de las guarniciones militares de fines del siglo XIX, ubicadas en el interior de la pampa seca.

Palabras clave: asentamiento militar, pipa artesanal, microvestigios vegetales, prácticas fumatorias.

\begin{abstract}
In this article we present the results obtained from the analysis of microvestiges of plants identified in an artisanal pipe found in Fort La Perra, Loventue department, La Pampa. In this place a small military settlement was installed; it formed part of one forts line installed after 1882 that was an military strategy of the territory vigilance and control , after the Ranqueles Indians were expelled because the Conquest of the Desert in 1879. The settlement pattern of this site as well as the vitreous, faunal and recovered metal materials has been the subject of different studies already published. In this case, the specific study of the contents of the pipe constitutes a new contribution to the knowledge gained about the lack of provisions and the isolation to which the members of the military garrisons of the late nineteenth century, located inside the dry pampa.

Keywords: military settlement, artisan pipe, microvestiges of plants, smoking practices.

\footnotetext{
* Universidad de Buenos Aires, Facultad de Filosofía y Letras, Instituto de Arqueología y Universidad Nacional de Luján, Departamento de Ciencias Sociales: aliciahtapia@yahoo.com.ar

** Universidad de Buenos Aires, Facultad de Filosofía y Letras, Instituto de Arqueología: virpineau@gmail.com

*** Becaria CONICET. Universidad Nacional de La Plata, Facultad de Ciencias Naturales y Museo: Laboratorio de Análisis Cerámico: meli_aug47@hotmail.com
} 


\section{Introducción}

A partir de 1882se estableció una línea de asentamientos militares en la pampa central extendida a lo largo de $150 \mathrm{~km}$ entre dos fortines cabecera de brigada, ubicados en las actuales localidades de Victorica y General Acha en la provincia de La Pampa (Memoria de Guerra y Marina, 1883-1884). La misión que debían cumplir las tropas acantonadas en tales asentamientos fue la de ejercer control y vigilancia, con el afán disuasorio de evitar cualquier tipo de resistencia de los ranqueles -que aún persistían en grupos pequeños y aislados en el norte pampeano- y de esa manera afianzar la ocupación del territorio después de la Conquista del Desierto en 1879. Si bien el funcionamiento de los fortines cabecera perduró hasta 1885, cuando se produjo la retirada definitiva del ejército del territorio nacional de La Pampa, el resto de los asentamientos militares que integraban esa línea tuvieron una existencia más efímera; ya sea por la falta de objetivos específicos que cumplir como por la falta de comunicación y de recursos necesarios para la subsistencia de las guarniciones (Raone, 1969).

El Fortín La Perra (FLP) constituye un ejemplo de este último caso, que al igual que otros asentamientos militares de su tipo incluían unos pocos integrantes(Figura 1). Fue instalado en la cima de un cerro de tosca de 349m.s.n.m, a una distancia de $75 \mathrm{~km}$ de ambos fortines cabeceras y a escasa distancia de una laguna conocida con el topónimo mapudungun Trehua lauquen (laguna del perro). Las particularidades del registro arqueológico de dicho sitio, tales como el patrón de asentamiento, los artefactos de vidrio, de metal y los restos faunísticos, han sido explicitadas en diversos trabajos ya publicados (Doval y Tapia, 2013; Pineau y Spota, 2007; Landa, 2011; Tapia y Pineau, 2004; entre otros)

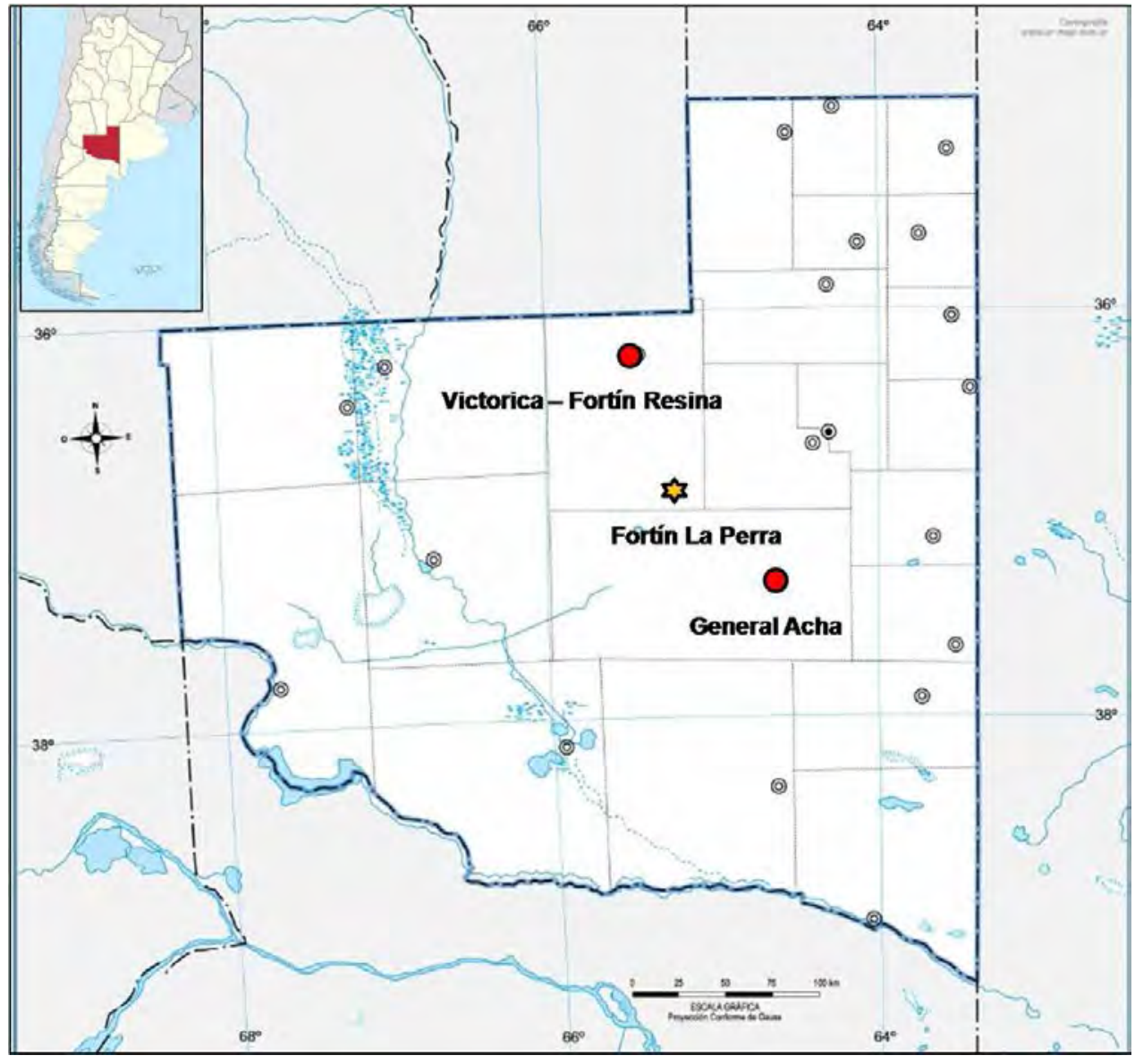

Figura 1. Ubicación del Fortín La Perra en la provincia de La Pampa y de los Fortines cabecera en las localidades de Victorica y General Acha. 
Resulta de interés considerar los resultados obtenidos a partir del estudio de los restos faunísticos recuperados en FLP (Doval y Tapia, 2012, 2013). La composición taxonómica de la muestra indica que el consumo de especies silvestres supera al de las especies domesticadas, hecho que puede estar vinculado con una escasa frecuencia del aprovisionamiento de ganado ovino y vacuno, que por reglamentación debía enviarse a los destacamentos militares para subsistencia de las tropas. Esta argumentación se ve reforzada por la identificación de huellas de procesamiento en restos óseos de Equus caballus: dado que este animal era imprescindible para la vida en la campaña, quizá fue consumido bajo una situación de muerte accidental del animal o bien por estrés alimenticio.

Por otra parte, tanto el estudio morfológico y funcional de 18 vainas Calibre 43 (11,5 x $58 \mathrm{~mm}$ ) de armas Remington Patria encontradas en el sitio como los datos que se mencionan en algunos documentos escritos, dan cuenta de la existencia de irregularidades en la provisión de alimentos, armas y proyectiles (Memoria de Guerra y Marina, 1882-1885; Tapia, De Rosa y Landa, 2009). En el caso de las vainas de FLP se observó que el 45 \% no presenta marca de percusión en el culote o rim, lo cual indica que no fueron utilizadas en disparos. Sin embargo están vacías, sin la pólvora y sin el correspondiente proyectil de plomo. En parte, algunas de ellas pudieron ser usadas para obtener la pólvora y encender el fuego, en tanto que otras pudieron ser descartadas por quedar atascadas en el interior de las armas que no funcionaban adecuadamente, ya sea porque estaban sucias o tenían los mecanismos deteriorados. Si bien el estado debía proveer al ejército nacional de todos los suministros -tanto armas y proyectiles producidas en el Parque de Artillería como raciones de comida, tabaco, etc. por medio de proveedores contratados-, el periodo de entrega y distribución no se cumplía adecuadamente. En las fuentes escritas se denuncian casos de corrupción, fraude y soborno. En tal sentido Álvaro Barros expresó: “(...) el proveedor que se llevaba bien con el jefe entrega efectos de pésima calidad y obtiene recibo superior y este recibo es confeccionado con arreglo a las listas de revista, y no al consumo verdadero (Barros, 1975 [1872], p104).

En este trabajo, con el objetivo de determinar el tipo de vegetales que podrían haber sido utilizados en las prácticas fumatorias de los integrantes de FLP, se analizan los microrrestos vegetales identificados en una pipa artesanal recuperada en una de las unidades de excavación. Los resultados obtenidos proporcionan un nuevo aporte a los conocimientos alcanzados sobre la falta de provisiones y el aislamiento al que fueron sometidos los integrantes de las guarniciones militares ubicadas en el interior de la pampa seca a fines del siglo XIX, y de qué manera esos factores impactaron en sus prácticas sociales de supervivencia.

\section{Características de la pieza y técnicas arqueométricas utilizadas}

La pieza fue confeccionada con el cuerpo de una vaina Calibre 43 de armas Remington Patria, utilizadas por el Ejército argentino entre 1873 y 1891 y luego reemplazadas por los fusiles y carabinas Mauser como armas reglamentarias. Los análisis arqueométricos previos de microscopía óptica, microscopia electrónica de barrido (SEM) y de energía dispersiva de rayos X (EDX) -realizados para identificar la estructura metalográfica- han permitido corroborar que la materia prima utilizada en su confección fue el latón, un metal típico con el cual se confeccionaron los proyectiles para armas largas durante la segunda mitad del siglo XIX. Dado que la base del proyectil no presenta el hoyuelo típico del percutor de disparo, se trata de una vaina que no fue disparada, pero se desconoce cuál pudo haber sido el uso que se le dio a la pólvora extraída (Tapia et al., 2009).

Para confeccionar la pipa se procedió a recortar la vaina a la altura en que comienza el cuello de la pieza (1/4 del largo) y como herramienta cortante se habría utilizado un formón cuyo filo dejó rastros de 
corte biselado (Figura 2). El fragmento inferior de la vaina fue utilizado como hornillo y con el fin de adosarle una boquilla se le efectuó un orificio cercano a la base o rim. La boquilla fue confeccionada con una diáfisis de hueso largo, que probablemente corresponde a una tibia de Ardea sp. (garza blanca) y que se unió al cuerpo de la vaina utilizando un fragmento de tela que aún se encuentra adherida a la abertura y a un sector de la pared de la vaina. Es posible que al encender el hornillo resultara difícil sostener la pieza con la mano por la elevada temperatura trasmitida a través del metal. Por este motivo, parte de la tela que sellaba la unión del metal con el hueso habría sido utilizada como aislante. Asimismo, en el registro arqueológico de FLP se recuperaron dos fragmentos correspondientes a la porción superior de dos vainas recortadas, coincidentes con el tipo de rastro de corte que se observa en el borde superior de la pipa y un fragmento de tibia de Ardea sp. con cortes transversales similares a los observados en el tubo de la pipa. De acuerdo con ello, es probable que la pipa recuperada en forma completa no sea la única pieza de ese tipo y que los fragmentos descartados indiquen la presencia de un número mínimo de al menos dos pipas similares.
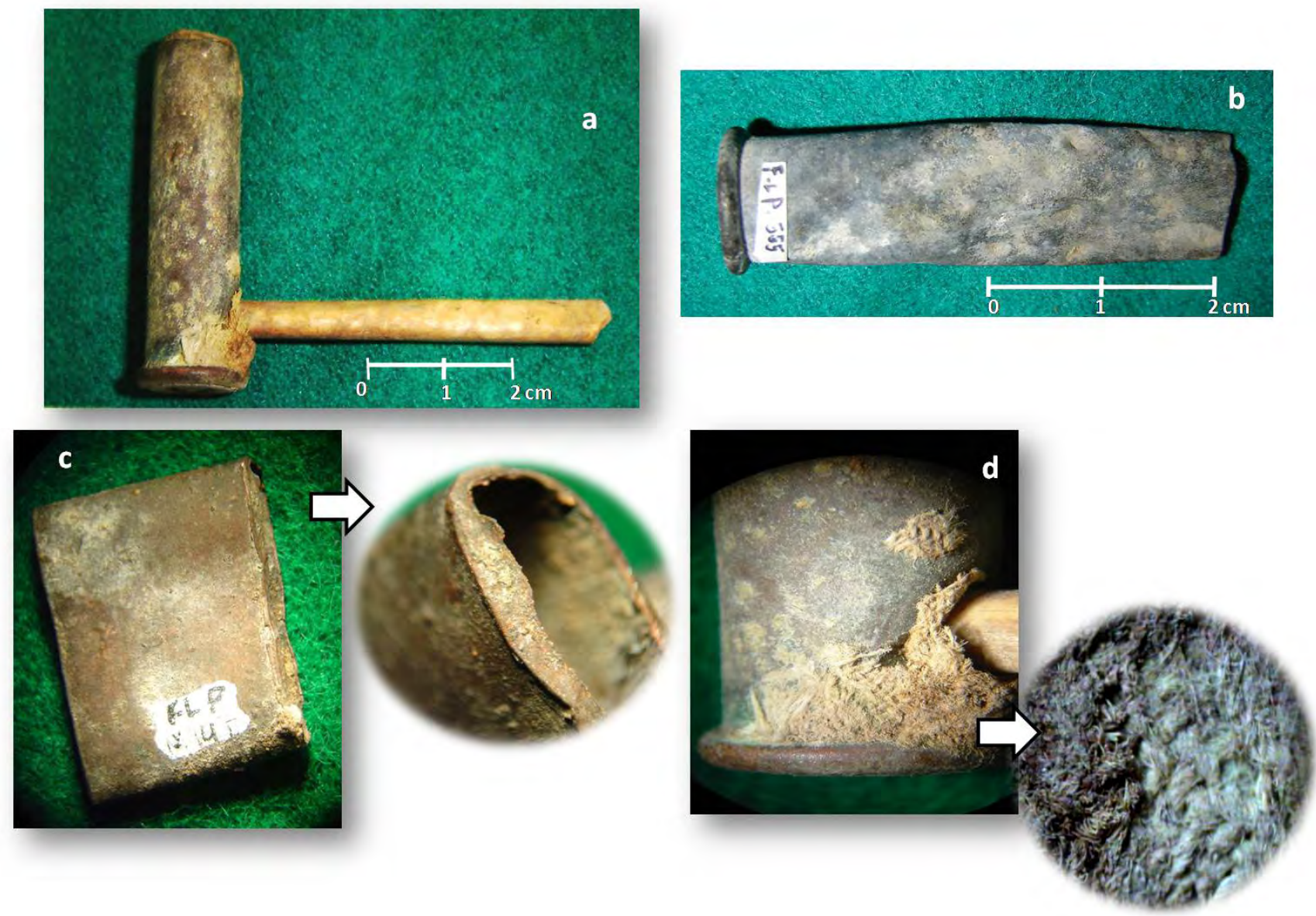

Figura 2. Características de la pipa artesanal hallada en FLP: a- artefacto completo; b- vainas C43 de armas Remington Patria sin la bala de plomo; c-corte de la vaina realizado en bisel con formón; y dtela utilizada para asegurar la inserción del tubo de hueso al orificio de la vaina. 
El material del interior de la pieza fue analizado por una de las coautoras en el Laboratorio 129 de la División de Arqueología de la Facultad de Ciencias Naturales y Museo de la Universidad Nacional de La Plata, dirigido por la Dra. Aylén Capparelli. La recuperación de residuos de la pipa se realizó siguiendo el protocolo propuesto por Lema et al. (2015) y testeado por el equipo de investigación en distintas oportunidades (Andreoni, Spano y Lema, 2012; Auge, Andreoni y García Lerena, 2016, 2018a). El mismo consiste en el raspado directo de las paredes internas del hornillo y del tubo. También se efectuó el tratamiento químico de las muestras con ácido láctico durante tiempos variables de exposición para optimizar la visibilidad en el Microscopio Óptico Leica MDLM (M.O.) bajo aumentos de 20x y 50x, con luz normal y polarizada.

Se obtuvieron cuatro muestras: relleno del tubo (M1); raspado interno del hornillo (M2); relleno del hornillo (M3) considerada una muestra control de sedimento; y material textil (M4) que al no estar quemado no se trató con ácido láctico sino que se realizó una observación directa con montaje en aceite de inmersión. Cabe aclarar que en todos los casos se usó material nuevo descartable y, previamente a la realización de cada tarea se lavaron las manos del operario, se limpiaron las superficies de trabajo y se tomaron las precauciones necesarias para evitar contaminaciones.

Los resultados del análisis de las muestras arqueológicas fueron comparados con una colección de referencia que incluye especies silvestres y cultivadas de uso fumatorio mencionados en distintas fuentes documentales y orales. Se recopilaron descripciones bibliográficas para Nicotianas sp. cultivadas y silvestres (Goodspeed, 1954), y se consultaron trabajos descriptivos de diferentes especies del género Nicotiana (Albornoz, 2015; Quiroz, Belmar, Planella, Niemeyer, Albornoz y Meneses, 2015) y de Ilexparaguariensis A. St. Hil. (Amat, 1991). Además se consultaron trabajos que presentan evaluaciones sobre la conservación de elementos diagnósticos de Nicotiana sp., después de los procesos de fumado (Auge et al, 2018b).

\section{Resultados obtenidos}

El análisis de los residuos de la pipa permitió reconocer estructuras de origen vegetal y animal, algunas de las cuales pudieron ser identificadas (ver Tabla 1 y Figura 3). En las muestras M1, M2 y M3se observaron elementos correspondientes a órganos foliares de Ilexparaguariensis A. St. Hil. (yerba mate). Algunas estructuras que de forma aislada carecen de valor diagnóstico por su redundancia en el Reino vegetal, como tricomas unicelulares simples o fragmentos epidérmicos, también podrían ser asignadas a Ilexparaguariensis teniendo en cuenta el contexto. Por su parte, M4 se compone íntegramente de fibras asignables a Gossypium sp. (algodón). La presencia de estas últimasestructuras vegetales en M1 y M2indicaría el desprendimiento de fragmentos desde eltextil ubicado en el punto de unión del hornillo y el tubo.

En todos los casos, el conjunto de elementos observados indica el consumo de partes foliares (epidermis, elementos conductores, tricomas, estomas). Asimismo, la presencia de estructuras que no se corresponden a I. paraguariensis a Gossypium sp. puede estar indicando el uso de más de una planta en las prácticas de fumado. El relleno del hornillo (M3) consistía en cenizas mezcladas con sedimento. Por esto, a pesar de considerarse como una muestra control (ya que es la parte de la pipa con más posibilidades de entrampar sedimento del suelo), las estructuras presentes en M3 con evidencias de termoalteración fueron consideradas como residuos integrantes de la práctica fumatoria. 
Teoría y Práctica de la Arqueología Histórica Latinoamericana | Año VIII. Vol. 9 | 2019

ISSN en línea: 2591-2801 | ISSN versión impresa: 2250-866X

Tabla 1. Resultados del análisis de microrrestos vegetales y determinacionestaxonómicas realizadas.

\begin{tabular}{|c|c|c|}
\hline Muestra & Resultados & Asignación taxonómica \\
\hline & Tejido epidérmico. & Indeterminado. \\
\hline & Cristales cuadrangulares a rectangulares. & Inderminado. \\
\hline & Hueso (Figua 3 a). & $\begin{array}{l}\text { Materia prima del tubo de la } \\
\text { pipa. }\end{array}$ \\
\hline \multirow[t]{4}{*}{ M1 } & Tejido epidérmico estriado, con estomas (Tigua 3 b). & Ilexparaguariensis A. St. Hil. \\
\hline & Tricoma unicelular (Figura 3 c). & Indeterminado. \\
\hline & Cristales cuadrangulares a rectangulares (Figura 3 d). & Indeterminados. \\
\hline & Fragmento de tejido, termoalterado. & Indeterminable. \\
\hline \multirow[t]{2}{*}{ M2 } & $\begin{array}{l}\text { Tejido epidérmico con presencia de estomas (Figura } 3 \\
\text { e). }\end{array}$ & Ilexparaguariensis A. St. Hil. \\
\hline & Fragmento de fibra (Figura $3 \mathrm{f}$ ). & afín a Gossypiumsp. \\
\hline
\end{tabular}



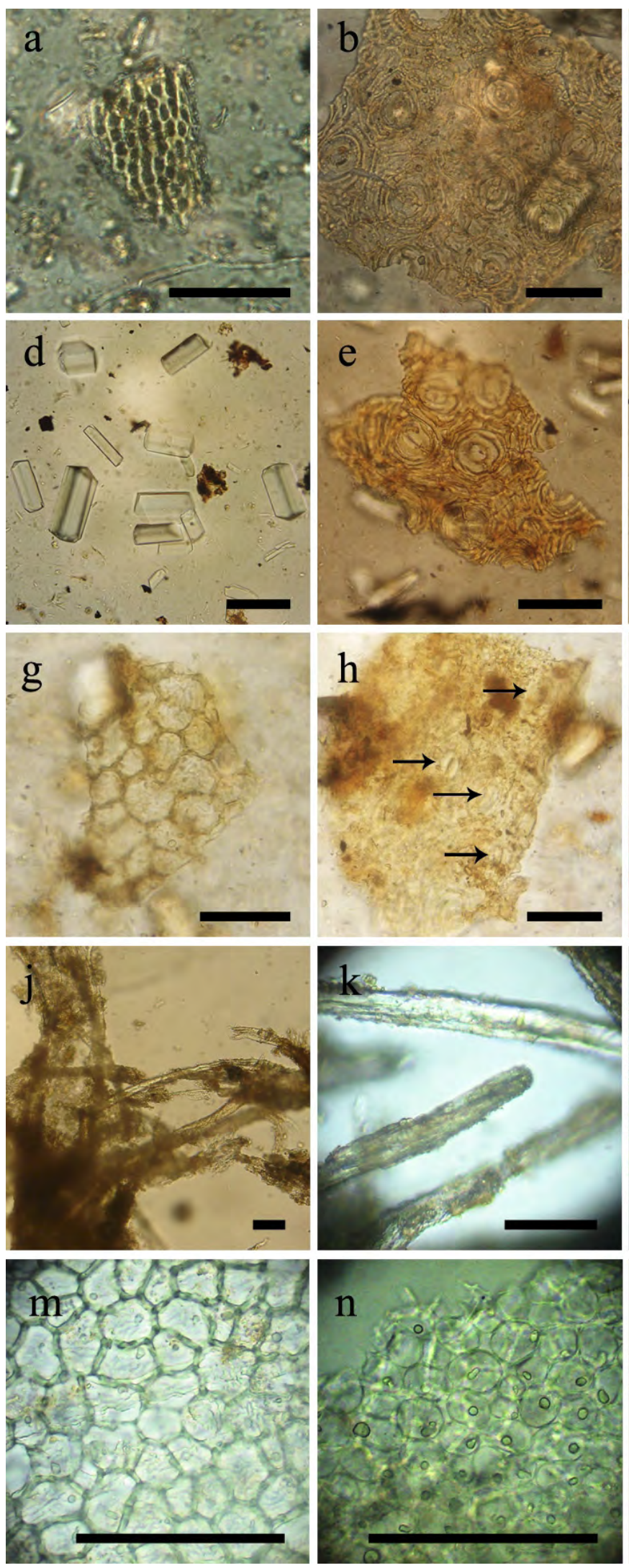
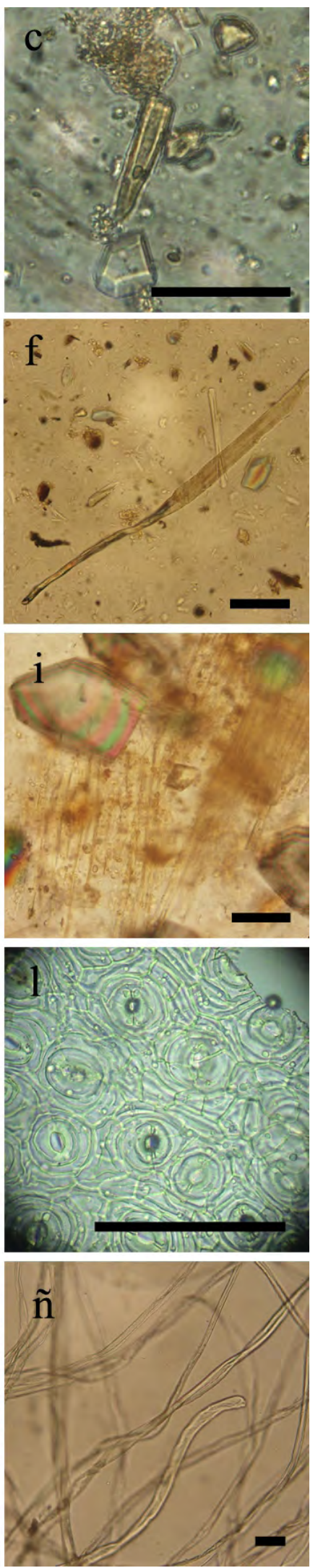

Figura 3. Material arqueológico: a- fragmento óseo; b- epidermis con estriación y estomas afín a Ilexparaguariensis A. St.Hil; c- tricoma unicelular; d- cristales; e- epidermis con estriación y estomas afín a Ilexparaguariensis A. St. Hil; f- fibra plana, gira sobre sí misma, puntas redondeadas, afín a Gossypium sp.; g- epidermis con células poligonales de pared engrosada, sin estriaciones, afín a Ilexparaguariensis A. St. Hil; h-epidermis, con estriación y estomas afín a Ilexparaguariensis A. St. Hil (se señalan los estomas); i- tejido indeterminado; j-k fibras planas, rotan sobre sí mismas, extremos redondeados, afines a Gossypium sp. Material de referencia: 1- epidermis abaxial; $\mathrm{m}$ - epidermis adaxial; $\mathrm{y}$ n-mesófilo de Ilexparaguariensis A. St.Hil; ñfibras de Gossypium sp. (escala: 50 um). 
Teoría y Práctica de la Arqueología Histórica Latinoamericana | Año VIII. Vol. 9 | 2019

ISSN en línea: 2591-2801 | ISSN versión impresa: 2250-866X

\section{Discusión y Conclusiones}

Teniendo en cuenta que la especie vegetal identificada como principal componente utilizado para fumar no corresponde a tabaco ( $N$. tabacum), se infiere que los integrantes de la guarnición militar estuvieron sometidos a una baja frecuencia en el envío de raciones de ese artículo, hecho que se habría acentuado por la distancia a la fuente de aprovisionamiento. Esta argumentación se correlaciona con los datos del registro faunístico analizado previamente, los cuales indican un significativo consumo de fauna silvestre en contraste con el ganado domesticado. Para sortear las marcadas condiciones de aislamiento y escasez de recursos, los integrantes del fortín habrían recurrido a la existencia de sus saberes tradicionales sobre hierbas con propiedades aptas para encender y aspirar el humo. Al respecto, resulta de interés señalar algunas menciones que se encuentran en la bibliografía sobre el uso fumatorio de Ilexparaguariensis entre los tehuelches. Gancedo (1973), parafraseando a Musters, menciona que los indígenas conseguían tabaco en las colonias pero que cuando este escaseaba: consiguen de los araucanos una hierba sucedánea. Esta hierba se utiliza mezclada con madera triturada o con tallos de yerba mate (Gancedo, 1973, p. 49). Según narra Mansilla, los ranqueles también consumían una especie de tabaco que le proporcionaban los “indígenas chilenos” y que él mismo fumó, resultándole muy fuerte y con efectos narcóticos muy parecidos al opio (Mansilla, 1993). En el área fitogeográfíca del caldenal o el espinal -donde se ubica el sitio Fortín La Perra- se ha identificado una planta solanácea denominada Nicotiananoctiflora Hook. Varnoctiflora conocida vulgarmente como "tabaco cimarrón” (aukpetrem en mapudungun), sin embargo a pesar de su nombre resulta tóxica y fétida, siendo bastante poco probable que fuera utilizada para fumar.

Respecto del caso que se analiza, los resultados obtenidos no permiten descartar la posibilidad que se hayan usado otros vegetales para fumar, además de la yerba mate. Atendiendo a la mención que realiza Musters sobre el caso tehuelche, la yerba mate bien puede haber sido utilizada como complemento para preservar por más tiempo el encendido, dado que contiene tallos más resistentes al fuego a diferencia del picado fino de hojas, ya sea de tabaco como de otras especies de hierbas fumatorias. No obstante, en el análisis de los microrrestos vegetales recuperados en la pipa del Fortín La Perra no se identificó la presencia de tallo de yerba mate sino solo componentes de la hoja. En la medida que el uso de yerba mate pudo haber reemplazado al tabaco en algunas ocasiones, la práctica registrada en FLPno constituye un caso aislado y atípico sino una práctica fumatoria que habría persistido como saber tradicional entre indígenas y criollos, contemporáneamente al uso de pipas industriales y al consumo de tabaco elaborado comercialmente.

En la documentación escrita (listas de aprovisionamiento, informes militares y administrativos, etc.), entre las provisiones que se enviaban para abastecer a los diferentes establecimientos militares ubicados en las líneas fronterizas de las subregiones pampa húmeda y pampa seca, siempre se menciona el envío de tabaco definido como "vicio", junto con la yerba mate. Si bien el tabaco estaba destinado a toda la guarnición, su distribución entre los integrantes de la tropa debió estar racionada y, de la misma manera que el resto de los vicios, habría sido utilizado por la oficialidad para premiar o castigar. La carencia de raciones de este artículo que debería haber enviado el Ejército, el aislamiento y la necesidad de apaciguar la soledad, habrían incentivado el uso de la yerba mate para fumar como una práctica no desconocida en el imaginario de los ocupantes del fortín. Por el contrario, resulta muy ingeniosa y creativa la confección de la pipa que reúne materiales naturales como el fragmento de tibia de ave y materiales de fabricación industrial como la vaina y el fragmento de tela de algodón. 
Teoría y Práctica de la Arqueología Histórica Latinoamericana | Año VIII. Vol. 9 | 2019

ISSN en línea: 2591-2801 | ISSN versión impresa: 2250-866X

\section{Referencias bibliográficas}

ALBORNOZ, X. (2015). Colección de referencia de elementos histológicos para estudios de microrrestos vegetales: especies psicoactivas y aromáticas de los Andes Centro-Sur. En

C. Belmar y V. Lema (Eds.), Avances y desafíos metodológicos en arqueobotánica: miradas consensuadas y diálogos compartidos desde Sudamérica (pp. 497-516). Santiago de Chile, Chile: Universidad SEK.

AMAT, A. G. (1991). Caracteres histofoliares diferenciales de Ilexparaguariensis St. Hil. (“yerba mate”) y su adulterante Mangifera indica L. (“mango”). Acta Farmacéutica Bonaerense,10 (1), 9-13.

ANDREONI, D., SPANO, R., y LEMA, V. (2012). Nota sobre evidencias de uso de plantas en el sitioSoria 2 a partir del análisis microscópico del contenidode pipas. Arqueología,18, 235-243.

AUGE, M., ANDREONI,D., Y GARCÍA LERENA, M. S. (2016). Una aproximación al uso de plantas a partir del análisis de microrrestos vegetales en pipas de caolín del sitio Estancia Bertón (Magdalena, Buenos Aires).En Actas del XIX Congreso Nacional de Arqueología Argentina, Serie Monográfica y didáctica Volumen 54 (pp. 28-34). San Miguel de Tucumán, Argentina: Publicación de la Facultad de Ciencias Naturales e Instituto Miguel Lillo de la Universidad Nacional de Tucumán.

AUGE, M., ANDREONI, D., y GARCÍA LERENA, M. (2018a). El uso de plantas como fumatorios en los partidos de Magdalena y Punta Indio (Buenos Aires): un análisis de microrrestos vegetales en pipas arqueológicas de la ribera rioplatense. Interseccionesen Antropología,19(2), 145-157.

AUGE, M., ANDREONI, D., y GARCÍA LERENA, M. (2018b). Las prácticas fumatorias en el litoral rioplatense desde una perspectiva arqueológica experimental: una evaluación de la conservación de elementos anatómicos diagnósticos de Nicotiana. Revista de Antropología del Museo de Entre Ríos,4(2), 29-40.

BARROS, A. (1975 [1872]). Fronteras y territorios federales de las Pampas del Sur. Buenos Aires, Argentina: Editorial Hachette.

DOBRIZHOFFER, M. (1967). Historia de los Abipones, Volumen I. Resistencia, Chaco, Argentina: Universidad Nacional del Nordeste, Facultad de Humanidades, Departamento de Historia.

DOVAL, J., y TAPIA, A. (2012). ¿Basural o acumulación natural de restos faunísticos? Fortín La Perra (1883-1885), La Pampa. Comechingonia, 16(2), 9-30.

DOVAL, J., y TAPIA, A. (2013). Consumo de fauna domesticada y silvestre en el Fortín La Perra (18831885), La Pampa. Revista Relaciones de la Sociedad Argentina de Antropología,38(1 y 2), 459492.

GANCEDO, O. (1973). Descripción de las pipas de fumar tehuelches de la Colección Francisco P. Moreno y Estanislao Zeballos, Revista del Museo de La Plata,(Nueva Serie) VI, I Antropología, 51, 47-55.

GOODSPEED, T. (1954). Thegenus Nicotiana. Waltham, Mass, U.S.A: Chronica Botanica Company. 
LANDA, C. (2011). Fierros fronterizos: los materiales de metal como indicadores de identidad y diferenciación social en la Frontera Sur (1776-1885). Madrid, España: Editorial Académica española.

LEMA, V., ANDREONI, D., CAPPARELLI, A., ORTIZ, G., SPANO, R., QUESADA, M. y ZORZI, F. (2015). Protocolos y avances en el estudio de residuos de pipas arqueológicas de Argentina. Aportes para el entendimiento de metodologías y prácticas pasadas. Estudios Atacameños, 51, 77-97.

MANSILLA, L. (1993). Una excursión a los indios ranqueles. Buenos Aires, Argentina: Colección Austral, Espasa Calpe.

MEMORIA DE GUERRA Y MARINA (1983-1985). Tomo I. Buenos Aires, Argentina: Archivo General de la Nación.

PINEAU, V., y SPOTA J. C. (2007). Un embotellamiento en el desierto. Identificación de los tipos de bebidas consumidas y su posible procedencia en el Fortín La Perra (Pcia de La Pampa). En C. Bayón, A. Pupio, M., I. Gonzçalez. N. Flegengeimer y M. Frère (Eds.), Arqueología de Las Pampas, Tomo II (pp. 835-844). Buenos Aires, Argentina: Sociedad Argentina de Antropología.

QUIROZ, L., BELMAR, C., PLANELLA, M., NIEMEYER, H. M., ALBORNOZ, X. y MENESES, F. (2015). Colección de referencia de microrrestos de lasespecies nativas del género Nicotiana en Chile. EnC. Belmar y V. Lema (Eds.), Avances y desafíos metodológicos en arqueobotánica. Miradas consensuadas y diálogos compartidos desdeSudamérica, (pp.517-531). Santiago de Chile, Chile: Universidad SEK.

RAONE, J. (1969). Fortines del desierto, mojones de la civilización. Tomo III. Buenos Aires, Argentina: Biblioteca del suboficial.

TAPIA, A. y PINEAU, V. (2004). Materiales vítreos y descarte diferencial. Comparación entre una ocupación aborigen y otra militar de fines del siglo XIX. En G. Martínez, M. Gutiérrez, R. Curtoni, M. Berón y P. Madrid (Eds.), Aproximaciones contemporáneas a la arqueología pampeana. Perspectivas teóricas, metodológicas, analíticas y casos de estudio(pp. 387-401). Olavarría, Provincia de Buenos Aires, Argentina: Universidad Nacional del Centro, Facultad de Ciencias Sociales.

TAPIA, A., DE ROSA, H., y LANDA, C. (2009). Rastros de uso, reciclamiento y descarte en vainas C43 de Remington Patria (1879-1885, La Pampa). En A. Austral y M. Tamagnini (Eds.), Problemáticas de la Arqueología Contemporánea. Tomo III (pp. 455-466). Rio Cuarto, Córdoba, Argentina: Editorial de la Universidad Nacional de Río Cuarto.

Recibido: 19 de mayo de 2019

Aceptado: 4 de julio de 2019 